\title{
DESIGUALDADE SOCIOECONÔMICA NA SAÚDE PÚBLICA BRASILEIRA E SUA INFLUÊNCIA NO DESENVOLVIMENTO DE TRANSTORNOS MENTAIS
}

\author{
SOCIOECONOMIC INEQUALITY IN BRAZILIAN PUBLIC HEALTH AND ITS \\ INFLUENCE ON THE DEVELOPMENT OF MENTAL DISORDERS
}

\begin{abstract}
Adriana Souza da Silva ${ }^{1}$
RESUMO: A saúde mental é termo complexo que envolve inúmeras circunstancias do contexto psicossocial. E a desigualdade socioeconômica é um fator de risco que podem desencadear prejuízos na qualidade de vida e diretamente ligado a influência de desenvolvimento de transtorno mentais. Por esse motivo o presente trabalho busca desempenhar de forma ampla as características psicológicas e sociais que atualmente tem influencia para desencadear transtornos mentais entre os indivíduos. A pesquisa biográfica foi desenvolvida através de materiais digitais, artigos científicos indexados a plataforma reconhecidas no meio acadêmico. Com esse objetivo de reflexão e ampliação dos conhecimentos científicos na temática de saúde mental e correlação das desigualdades socioeconômicas dentro da saúde pública brasileira é imprescindível para ampliação e construção de políticas públicas de qualidade que possam alcançar um grande número de indivíduos no Brasil.
\end{abstract}

Palavras-chave: Saúde mental. Socioeconômica. Saúde pública. Vulnerabilidade social. Transtornos mentais.

ABSTRACT: Mental health is a complex term that involves numerous circumstances in the psychosocial context. And socioeconomic inequality is a risk factor that can impair quality of life and is directly linked to the influence of the development of mental disorders. For this reason, the present work seeks to broadly play the psychological and social characteristics that currently have an influence on triggering mental disorders among individuals. The biographical research was developed through digital materials, scientific articles indexed to a recognized platform in the academic world. With this objective, reflection and expansion of scientific knowledge on the subject of mental health and correlation of socioeconomic inequalities within Brazilian public health is essential for the expansion and construction of quality public policies that can reach a large number of individuals in Brazil.

Keywords: Mental health. Socioeconomic. Public health. Social vulnerability. Mental disorders.

${ }^{\text {I }}$ Possui graduação em PSICOLOGIA pela Faculdade de Educação e Meio Ambiente - FAEMA. Pós- graduanda em saúde pública com ênfase em ESF. Atualmente residente em Saúde da Família e Comunidade no município de Vilhena/RO pela Faculdade de Educação e Cultura Vilhena - UNESC. 


\section{INTRODUÇÃO}

O acesso aos serviços de saúde brasileiro ocorreu através do desenvolvimento dos princípios e diretrizes do Sistema Único de Saúde (SUS), apresentando de forma integra e excepcional as bases teóricas e premissas para desempenhar um processo adequado a saúde da população. Mas, antes do desenvolvimento do (SUS) é possível ressaltar que o Brasil é um país subdesenvolvido, onde as condições socioeconômicas perpassam a cada ano por dificuldades diferentes e intensas entre a população (ALMEIDA; ATHAYDE, 2015).

Para Organização Mundial de Saúde (OMS) o termo saúde é compreendido como um estado completo e harmônico de bem-estar físico, mental e social para o indivíduo e não apenas a ausência de doença. E diante dessa definição é possível notar a influência gigantesca dos aspectos ambientais e sociais na vida do ser humano, onde geralmente a causa/motivo para desencadeamento de patologias está envolvida neste cenário social. Mostrando que a saúde está diretamente ligada com a conceito de qualidade de vida, no caso tal objetivo só pode ser alcançado pelo ser humano quando oferecido condições mínimas (ROCHA; LAPREGA, 20II).

E dentro do contexto social brasileiro apresentado, os transtornos mentais tem

ultrapassado as taxas de ocorrência na sociedade. Visto que a saúde mental em diversos âmbitos tem sido negligenciada, em modo individual, social e político. E os altos níveis de prevalência de transtorno mentais entre os indivíduos vêm provocando prejuízos ao sujeito, família, comunidade e sistema de saúde (OMS, 2008).

Para Gaino et al. (2018) a nova proposta relacionada ao olhar da conjuntura do processo de saúde, tem mostrado a necessidade de buscar um cuidado e análise para os diferentes fatores, entre eles educação, dieta, moradia, questões familiares, condições de trabalho, renda, lazer e meio social entre outros. Com isso, demostrando claramente os princípios do (SUS) sobre universalidade, integralidade e equidade, assim como a função essencial da equipe multidisciplinar em um trabalho coordenado para alcançar um atendimento adequado e acesso ao serviço de qualidade aos usuários. 
Desta maneira, o estudo busca demostrar aspectos socioeconômicos e os principais desafios na sociedade para que seja possível alcançar saúde de qualidade e concomitantemente saúde mental. Onde os conceitos teóricos possam sem estabelecidos no ambiente de prática do serviço de saúde. E apresentar a saúde pública brasileira a partir dos múltiplos envieses, que tem influência dentro do processo de saúde.

É importante ressaltar que pesquisas dentro dessa perspectiva têm a possibilidade de conduzir e estimular informação de qualidade e cunho cientifico para comunidade ligada aos estudos de saúde pública. Uma área de grande crescimento e mudanças sociais, visto que as novas tecnologias e ascensão da globalização transforma a sociedade diariamente. O serviço de saúde ocorre na forma prática pelas possibilidades de estudos teóricos que desenvolve senso crítico e especializado para os atores/protagonistas na saúde.

O presente artigo apresenta uma pesquisa bibliográfica realizada através das bases dados atuais, em artigos científicos, indexados a plataformas digitais como google acadêmico, BVS, Scielo, Pepsic, websait oficiais pertinentes a temática. E dessa forma, busca demostrar análise referente ao processo de saúde e a influência da desigualdade social como fator prejudicial para alcançar saúde mental adequada.

\section{SAÚDE PÚBLICA PERSPECTIVA BRASILEIRA}

Com a criação e consolidação da Constituição Federal Brasileira em 1988, onde ficou estabelecido no artigo 196 que a saúde é direito de todo cidadão e dever de o estado garantir através políticas públicas, sociais e econômicas de qualidade que vise a redução de danos e agravos de doenças, com acesso universal e igualitário, ações e serviços para promoção, proteção e recuperação da saúde (BRASIL, 20I6). O Sistema Único de Saúde nasceu com a intenção de fornecer a população serviço de saúde de qualidade e de forma gratuita, sem distinção alguma.

Ao longo dos anos é muito comum ouvir em meios de comunicação sobre os princípios e diretrizes do Sistema Único de Saúde (SUS) brasileiro. Onde esses princípios e diretrizes foram delineados pela Constituição Federal, destacando a universalidade equidade, integralidade, descentralização, participação da população e a organização da 
rede de serviços de modo regionalizado e hierarquizado. E ao analisar o processo histórico é possível compreender que o SUS foi desenvolvido a partir do movimento da Reforma Sanitária entre os anos 70 e 8o. Mas, só em 1990 o Congresso Nacional aprovou a lei Orgânica da Saúde vigente atualmente, e a partir desse momento foi assegurado o direito a saúde universal e gratuita a população. O SUS é administrado através dos recursos federais, estaduais e municipais, estabelecidos em forma de tripartite na Emenda Constitucional 29 (EC-29) no ano 2000 (MATTOS, 2009).

Dessa maneira, os princípios e diretrizes do SUS devem ser compreendidos na perspectiva da história e da epistemologia, pois é produto de um processo político e expressa conceitos sobre a relação entre saúde e doença, direitos sociais, gestão e campos do governo nacional. Destacando assim, os três princípios básicos do SUS: a Universalidade do SUS não se manifesta apenas na garantia do direito a saúde por meio de políticas públicas, como também olhar voltado ao direito a vida e igualdade de oportunidades, independente de raça, sexo, religião ou qualquer outra forma de discriminação as pessoas. Dentro da Equidade apresenta adaptação da regra existente à situação concreta, observando-se os critérios de justiça. Ou seja, equidade busca adaptar a regra a um caso específico, a fim de deixá-la mais justa. No âmbito do SUS é necessário direcionar os investimentos onde a equidade é maior, nas necessidades coletivas e individuais. E a Integralidade é definida como um conjunto claro de ações e serviços de saúde, que busque o tratamento individual ou coletivo seguindo a necessidade de caso, ao nível de complexidade do sistema (MATTA et al., 2007).

Em contra mão com os determinantes do SUS, o Brasil vem apresentando ao longo dos anos a grande tendência para distribuição desigual de renda entre a população, e concomitantemente desencadeando altos níveis de pobreza. Um país desigual vem ultrapassando por anos aspectos históricos para as injustiças sociais que exclui grande parte da população ao acesso as condições mínimas de cidadania e dignidade (BARROS; HENRIQUES; MENDONÇA, 200o). 
Dessa forma, demostrando que a saúde tem ligação direta com as condições socioeconômicas da população. Pois, identifica-se através contextos sociais definidos por fatores político e falta de administração pública a desigualdade estampada, assim como o crescimento de cidades desordenados sem planejamento, a falta de saneamento básico, falta água potável de qualidade, alimentação inadequada, condições precárias de moradia e trabalho entre outros fatores são determinantes no desenvolvimento de uma saúde de qualidade para as pessoas. E alguns indicadores de saúde como mortalidade infantil podem ser destacados como resultado direto da ausência de politicas públicas voltadas as pessoas em vulnerabilidade social (CAMPELLO et al., 2018).

Em consequência disso, a busca social por saúde pública de qualidade se consolida ao longo do tempo no Brasil. Vale ressaltar que a definição de saúde pública permeia entre a vinculação do aparelho do Estado em algumas decisões, assim como a dimensão coletiva como objeto de intervenção, manifestando autonomia popular. A saúde pública é um campo geral da prática e do conhecimento da organização institucional que visa realizar os ideais de bem-estar das pessoas em uma sociedade específica - em termos de ações e medidas para prevenir, reduzir e / ou minimizar os danos à saúde, garantir que os seres humanos sejam mantidos e manteve a vida. Por fim, pode-se inferir que a compreensão do que é "saúde pública", em última instância, advém da perspectiva de indivíduos / grupos socioeconômicos e culturais, restritos por suas visões sobre o mundo que nos rodeia, e com base em seus respectivos interesses, crenças e ideias. Porém, o mais importante é que seja determinado pela estrutura socioeconômica correspondente (FUNDAÇÃO OSVALDO CRUZ; PIRES FILHO, 1987).

\section{I Saúde mental e as principais influencia no atual contexto}

Em relação ao conceito de saúde mental é importante ressaltar por antemão o processo histórico do movimento da Reforma Psiquiátrica no Brasil. Destacado através dos anos 7o, onde o movimento nacional antimanicomial brasileiro tomou forças levantando a grande importância para a proteção dos direitos das pessoas com transtornos mentais, e sua principal característica foi o desaparecimento do asilo. Esse movimento social está relacionado às 
reformas da saúde no Brasil e enfatiza as discussões sobre os direitos humanos das pessoas com transtornos mentais (BARBOSA; COSTA; MORENO, 2012).

Por muitos anos a sociedade considerou a pessoa com transtorno mental um ser possuído por demônios, vitimas de punições divinas, pessoas preguiçosas, ruins ou loucas (DESVIAT; RIBEIRO, 2015). Com advento da reforma psiquiátrica possibilitou garantir, respeitar, proteger e humanizar os direitos sociais e, gradativamente, desconstruir a lógica da internação para o portador de transtorno mental (SILVA, 2014).

Com reformulação do cuidado e atendimento as pessoas com transtornos mentais houve dissonância entre a rede de saúde compondo um processo amplo para atender as diversas demandas, envolvendo atenção primária a saúde, o desenvolvimento dos centros especializados em saúde mental sendo Centro de Atenção Psicossocial - CAPS, alcançando quando necessário os atendimentos hospitalizados. Os Centros de Atenção Psicossocial são compostos por equipe multiprofissionais atuando de forma interdisciplinar. Onde as práticas terapêuticas são elaboradas dentro de ambiente acolhedor e aberto. Alcançando os diversos fatores que podem estar impedindo a reabilitação adequada do paciente, atingindo contexto familiar, social e até mesmo econômico (BERNARDI; KANAN, 2015).

De forma geral assim como apresentado anteriormente sobre o conceito de saúde para (OMS), a saúde mental não e diferente. A saúde mental não deve ser considerada apenas como ausência de algum tipo de transtorno mental, o conceito deve estar relacionado com compreensão muito maior. Pois, atualmente em algum momento as pessoas podem estar vulneráveis a vivenciar sentimentos ou emoções negativas que causem desconforto emocional. Onde o ser humano pode ter experiencia diariamente frente uma série de emoções como alegria, amor, tristeza, raiva entre outros. Dessa forma, estar com saúde mental adequada significa estar adaptado a enfrentar os desafios e mudanças do dia a dia de forma equilibrada, e sabem buscar ajuda no enfrentamento de conflitos, perturbações, traumas ou mudanças importantes nos diferentes ciclos da vida. A saúde mental de uma pessoa está relacionada à sua resposta às necessidades da vida e como ela coordena seus desejos, habilidades, ambições, pensamentos e emoções (OLIVEIRA; SOUSA, 2020). 
Segundo Gama, Campos e Ferrer (2014), a definição de saúde elaborada pela (OMS) se torna ultrapassada quando relacionada com a saúde mental, visto que o ser humano é rodeado de diversos contextos e fatores que podem estar influenciando em sua saúde. Dentro da existência de uma pessoa podem ser incluídos erros, fracassos, privações, as escolhas, desejos, angustias, desafios e contradições. E por isso, é muito importante que essa compreensão e definição perpasse através de uma conexão com a vida cotidiana e as singularidades. Além de subjetivo o conceito de saúde mental, é necessário que viver na atualidade é estar suscetível a diversos tipos de adoecimentos. Busca de nível adequado de saúde mental está muito relacionado a não romantização da vida, e sim a preparação de estratégias e mecanismos centrados na capacidade de enfrentar os problemas que possam ocorrer. E mesmo com desenvolvimento histórico da reforma psiquiátrica, e evolução cientifica existe muito preconceito por trás do diagnóstico psiquiátrico, pois além de estar vinculado com a situação do normal e patológico envolve uma discussão acerca da loucura e os estigmas relacionados.

Ao citar a desigualdade socioeconômica do Brasil automaticamente é relacionado com o conceito de vulnerabilidade social. E antes de explanar o tema é importante ressaltar que individuo não é remetido a imagem de vulnerável. Mas, o seu contexto externo/ambiental, ou seja, a vulnerabilidade está ligada a falta de acesso a bens materiais ou bens de serviços que posam suprir as necessidades principais (MARTINS et al., 2019).

Para o autor Abramovay et al. (2002) reforça a definição de vulnerabilidade social como ausência de recursos ou ferramentas de um grupo social se tornam insuficientes ou inadequadas para lidar com as oportunidades oferecidas pela sociedade. As oportunidades estão relacionadas as possibilidades de alcançar níveis maiores de bem-estar e qualidade de vida. Todavia, a vulnerabilidade social não se restringe apenas ao contexto econômico, mas um conjunto muito complexo de fatores, entre eles as organizações políticas, questão de raça, etnia, orientação sexual, gênero e cultura.

E dentro das questões já apresentadas, é importante relacionar que os determinantes sociais para saúde mental, não se limita apenas as questões de desigualdade social e pobreza. Demonstrando a evidencia e credito para a formulações de políticas públicas que facilite o 
acesso e qualidade aos serviços e ações de saúde pública e recursos comunitários, comparando ao grau de integração e suporte social. Apontando que desigualdade em saúde pode ser influenciadas por aspectos de condições de vida, recursos sociais, assistência à saúde, atual situação de saúde e questões culturais (DIMENSTEIN et al., 2017).

$\mathrm{Na}$ pesquisa desenvolvida por Alves e Rodrigues (2010), é apresentado de forma detalhada as causas determinantes no meio social e econômico para o desenvolvimento de transtornos mentais. No trabalho é ressaltado que o desemprego e insatisfação no trabalho é umas das grandes causas para aparecimento de doenças mentais. Outro fator importante são os níveis educacionais baixos, que concomitantemente resulta em oportunidades de emprego com remuneração baixa, condições de habitação inadequada e maior exclusão social. Onde os dois aspectos reunidos promovem altos níveis de pobreza, pois faz referencia a falta de dinheiro ou bens e serviços para necessidades básicas.

Também foram ressaltados na pesquisa a questão de habitação, pois uma casa confortável proporciona sensação de bem-estar. No caso da urbanização está refletida na superpopulação, estresse, ambiente poluído, enfraquecimento dos laços familiares, hábitos pouco saudáveis. Em contrapartida, ambientes isolados como zona rural pode propiciar o desenvolvimento de doenças psiquiátricas, através da falta de comunicação, falta de informação adequada, meios de transporte, acesso a educação e formação profissional, assim como o acesso aos serviços de saúde são limitados. Fato super relevante é a maior ocorrência de transtornos mentais entre as mulheres, possivelmente ocasionado por discriminação sexual, violência de gênero, violência doméstica, pouco autonomia, dificuldade no acesso a educação. As questões biológicas relacionado aos hormônios também podem influenciar, mas considera-se os aspectos psicológicos e sociais com maior influência, visto que atualmente a mulher desempenha várias funções na sociedade, com níveis altos de cobrança (ALVES; RODRIGUES, 2010).

Experiências precoces/ambiente familiar também sãs aspectos que podem ocasionar prejuízos na saúde mental, assim como gravidez na adolescência, presenciar na infância cenas de sexo, violência, uso de substâncias psicoativas como álcool, drogas ou tabaco, agressões 
físicas ou psicológica que podem prejudicar de forma intensa o desenvolvimento neurocognitivo. A discriminação, preconceito, racismo e estigmatização podem levar a exclusão social que está diretamente ligada a possibilidade de desenvolvimento de doenças mentais. A cultura é fator importantíssimo na saúde mental, pois algumas culturas possibilitam conjunturas familiares patológicas, a cultura pode reforçar alguns comportamentos desajustados como forma de aprovação social, também pode estabelecer práticas não saudáveis ou padrões rígidos de comportamento. E de forma geral os acontecimentos de vida estressante, diante da intensa evolução tecnológica as pessoas estão expostas a diversas dificuldades sejam familiares ou sociais, como exemplos de abuso sexual, violência em diversos contextos, situações de guerra, doenças crônicas, pandemias, epidemias, catástrofes naturais, onde todos os pontos mencionados podem desencadear transtornos mentais, elevando o risco de depressão, ansiedade, perturbações por estresse pós traumático e suicídio. Na pesquisa apresenta também que a problema contribui para prognostico negativo no quadro de doenças psiquiátricas, como também em muitos casos apresenta uma rede de apoio inadequada sem suporte familiar ou amigos. (ALVES; RODRIGUES, 2010).

Ao finalizar apresentação dos aspectos e fatores econômicos e sociais que podem influenciar em desenvolvimentos de transtornos mentais, para o autor Martins (2004) é de suma importância não desconectar do funcionamento biológico do ser humano visto que questão genéticas entre outras também podem influenciar. Portanto, os fatores biológicos podem interagir de forma integral juntamente com dimensão psicossocial do indivíduo referente sua saúde.

\section{CONCLUSÃO}

Em virtude dos fatos mencionados, compreende-se que a saúde mental deve ser analisada através de diversas circunstancias, visto que as experiências ao longo da vida, assim como ambiente externo e interno do indivíduo podem influenciar para desenvolvimento de transtornos mentais. Características biológicas e histórico pessoal e familiar estão diretamente 
ligados na qualidade da saúde mental. Ao mesmo tempo, que as condições socioeconômicas também podem influenciar tanto para progressão ou desencadear o processo de adoecimento no ser humano. onde essas variáveis psicológicas também podem ser denominadas através do termo psicossocial.

Por esse motivo é possível entender que a temática de saúde mental se torna complexa e até mesmo sendo um tema pouco explorado ou estudado, visto também certo preconceito social construído ao longo do tempo, sendo necessário disseminação de informação de qualidade para que a sociedade considere a saúde mental um fator primordial do ser humano.

Dessa forma, a desigualdade socioeconômica existente atualmente na sociedade, se torna um fator de risco desencadeador nos processos de adoecimento, como também dificulta um tratamento adequado, ou até mesmo acesso aos serviços essenciais e básicas ao ser humano, sendo considerado um limitante para um prognóstico favorável. E ampliação de conhecimento cientifico nesse contexto socioeconômico na saúde mental brasileira é ferramenta eficaz para proporcionar integração e formulação de políticas públicas numa perspectiva abrangente da saúde pública.

\section{REFERÊNCIAS}

ALMEIDA, A. R.; ATHAYDE, F. T. S. Promoção da saúde, qualidade de vida e iniquidade em saúde: reflexões para a saúde pública. Tempus Actas de Saúde Coletiva, v. 9, n. 2, p. ág. 165-172, 2015. Disponível em: https://www.tempusactas.unb.br/index.php/tempus/article/view/1818/1502. Acesso em: em I3 de jul. 2021.

ABRAMOVAY, M. et al. Juventude, violência e vulnerabilidade social na América Latina: desafios para políticas públicas / Miriam Abramovay et alii. - Brasília: UNESCO, BID, 2002. $192 \mathrm{p}$. 
ALVES, A. A. M.; RODRIGUES, N. F. R. Determinantes sociais e económicos da Saúde Mental. Revista Portuguesa de Saúde Pública, v. 28, n. 2, p. 127-131, 2010. Disponível em: https://www.sciencedirect.com/science/article/pii/So870902510700031. Acesso em: i4 de jul. 2021.

BARROS, R. P.; HENRIQUES, R.; MENDONÇA, R. Desigualdade e pobreza no Brasil: retrato de uma estabilidade inaceitável. Revista brasileira de ciências sociais, v. I5, p. I23-I42, 2000.

BARBOSA, G. C.; COSTA, T. G. D.; MORENO, V. Movimento da Luta Antimanicomial: Trajetória, Avanços e Desafios/The Anti-Asylum Fight Movement: Trajectory, Progress and Challenges. Cadernos Brasileiros de Saúde Mental/Brazilian Journal of Mental Health, v. 4, n. 8, p. 45-50, 2012.

BERNARDI, A. B.; KANAN, L. A. Características dos Serviços Públicos de Saúde Mental (Capsi, Capsad, Caps III) do Estado de Santa Catarina. Saúde em Debate, v. 39, p. IIo5-III6, 2015 .

BRASIL. [Constituição (1988)] Constituição da República Federativa do Brasil: texto constitucional promulgado em 5 de outubro de 1988, com as alterações determinadas pelas Emendas Constitucionais de Revisão nos I a 6/94, pelas Emendas Constitucionais nos I/92 a 9I/2016 e pelo Decreto Legislativo no r86/2008. - Brasília: Senado Federal, Coordenação de Edições Técnicas, 2016.

CAMPELLO, T. et al. Faces da desigualdade no Brasil: um olhar sobre os que ficam para trás. Saúde em Debate [online], v. 42, n. spe3, pp. 54-66, 2018. Disponível em: $\langle$ https://doi.org/10.1590/o103-11042018S305〉. ISSN 2358-2898. https://doi.org/ro.1590/o103II042018S305. Acessado I5 jul. 2021. 
DESVIAT, M.; RIBEIRO, V. A Reforma Psiquiátrica. SciELO-Editora FIOCRUZ, 2015.

DIMENSTEIN, M. et al. Determinação social da saúde mental: contribuições à psicologia no cuidado territorial. Arquivos Brasileiros de Psicologia, v. 69, n. 2, p. 72-87, 2017.

FUNDAÇÃO OSVALDO CRUZ. “O que é saúde pública?” [internet]. Biblioteca em saúde pública FIOCRUZ [data desconhecida]. Disponível em: http://www.fiocruz.br/bibsp/cgi/cgilua.exe/sys/start.htm?sid=I07. Acesso em: or agos. 202I.

Organização Mundial da Saúde. Constituição da Organização Mundial da Saúde (OMS/WHO) - 1946. Disponível em: 〈http://www.direitoshumanos.usp.br〉. Acesso em: 03 jul. 2021.

GAMA, C. A. P.; CAMPOS, R. T. O.; FERRER, A. L. Saúde mental e vulnerabilidade social: a direção do tratamento. Revista Latinoamericana de Psicopatologia Fundamental, v. I7, n. I, p. 69-84, 2014 .

GAINO, L. V. et al. O conceito de saúde mental para profissionais de saúde: um estudo transversal e qualitativo. SMAD Revista Eletrônica Saúde Mental Álcool e Drogas (Edição em Português), v. I4, n. 2, p. 108-116, 2018.

MATTA, Gustavo Côrrea et al. Princípios e diretrizes do Sistema Único de Saúde, EPSJV, 2007 .

MATTOS, R. A. Princípios do Sistema Único de Saúde (SUS) e a humanização das práticas de saúde. Interface-Comunicação, Saúde, Educação, v. 13, p. 771-780, 2009. 
MARTINS, M. I. F. et al. Saúde mental em contexto de vulnerabilidade social. Educação: Saberes e Prática, v. 8, n. I, 2019.

MARTINS, Maria da Conceição de Almeida. Factores de risco psicossociais para a saúde mental. Millenium, p. 255-268, 2004.

OLIVEIRA, A. L. X.; SOUSA, F. D. T. Saúde Mental: Um artigo de revisão sobre a Saúde Mental no Brasil. Revista Científica Multidisciplinar Núcleo do Conhecimento. Ano o5, Ed. 05, Vol. II, pp. 198-212. Maio de 2020. ISSN: 2448-0959. Disponível em:https://www.nucleodoconhecimento.com.br/saude/saude-mental-no-brasil.

ORGANIZAÇÃO MUNDIAL DA SAÚDE; ORGANIZAÇÃO MUNDIAL DE MÉDICOS DE FAMÍLIA. Integração da saúde mental nos cuidados de saúde primários: uma $\begin{array}{llll}\text { perspectiva } & \text { global. } & \text { em: }\end{array}$ 〈https://www.who.int/eportuguese/publications/Integracao_saude_mental_cuidados_primari os.pdf?ua=I>. Acesso em: 03 jul. 202I.

ROCHA, J. S. Y.; LAPREGA, M. R. Os determinantes sociais da saúde. Ibañez N, Elias P, Seixas P, organizadores. Política e Gestão Pública em Saúde. São Paulo: Hucitec, p. 219-4I, 2011.

PIRES FIlHO, F. M. O que é Saúde Pública?. Cadernos de Saúde Pública, v. 3, p. 62-70, 1987.

SILVA, Giselli Lucy Souza. A doença mental e a reforma psiquiátrica representadas por profissionais de saúde. 2014. 223 f. Dissertação (Mestrado em Psicologia Social) - Universidade Federal da Paraíba, João Pessoa, 20I4. 